\title{
Procesamiento de la información lingüística y lenguaje del pensamiento
}

\section{Processing of linguistic information and language of thought}

Mariela Destéfano*

\begin{abstract}
Resumen: En la psicología cognitiva del procesamiento de la información el lenguaje puede entenderse como un sistema de la mente/cerebro en el cual los outputs resultan de la aplicación de ciertas operaciones sobre representaciones a partir de ciertos imputs. En este trabajo quisiera argumentar contra la idea de que el lenguaje del pensamiento es el formato de las representaciones del procesamiento de la información lingüística. Primero, evaluaré los "argumentos del regreso" con los que se ha intentado sostener la tesis escéptica de este trabajo. Sostendré que estos argumentos no constituyen una buena estrategia para desechar al lenguaje del pensamiento como vehículo representacional. Segundo, desarrollaré una "inferencia a la explicación menos probable" con la que considero se puede poner en duda el rol del lenguaje del pensamiento como vehículo del procesamiento del lenguaje.
\end{abstract}

Palabras claves: representación mental, vehículo, comprensión lingüística, compiladores.

Abstract: In this article I will try to show that the Language of Thought (Lot) is not the vehicle of the representations involved in language processing. For that purpose, first, I will analyze the Regress Arguments, which are supposed to argue against Lot hypothesis. I will discard these arguments since they wouldn't be a good strategy. Second, I will present what I call an "Inference to the Least Likely Explanation" as a correct argument against Lot in language processing.

\footnotetext{
* Doctora en filosofía (UBA) especializada en la filosofía de la ciencia cognitiva. Se desempeña como docente en "Metafísica" (Carrera de Filosofía- UBA) y "Epistemología de las ciencias sociales" (Carrera de Sociología-UBA). También se desempeña como investigadora asistente (CONICET, Argentina) y como investigadora responsable en el proyecto: PICT-2014-3422 ("Las relaciones entre las arquitecturas cognitivas y las explicaciones en ciencia cognitiva”). Dirección electrónica: mariela.destefano@gmail.com
} 
Key words: mental representation, vehicle, linguistic comprehension, compilators.

\section{Introducción}

La psicología cognitiva del procesamiento de la información intenta dar respuesta a un subconjunto específico de preguntas-cómo. Intenta responder a cómo funcionan ciertos sistemas cognitivos. ${ }^{1}$ ¿Cómo el sistema visual produce un percepto? ¿Cómo el sistema lingüístico genera representaciones lingüísticas que serán ulteriormente utilizadas por otros sistemas cognitivos? Las explicaciones en este marco adoptan la forma de modelos en los que se especifican procesos y representaciones mentales. ${ }^{2}$ Desde esta perspectiva, el lenguaje puede entenderse como un sistema de procesamiento de la información en el sentido de que es un sistema de la mente/cerebro en el cual los outputs resultan de la aplicación de ciertas operaciones a partir de ciertos inputs. En tanto sistema de procesamiento, el lenguaje tendría ciertas limitaciones de memoria (no podría producir como output infinitos ítems) y estaría sujeto a posibles errores en el desarrollo de las operaciones. ${ }^{3} \mathrm{El}$ procesamiento temprano de la información lingǘstica puede entenderse que involucra una serie de componentes que van desde el estímulo auditivo hasta un output subpersonal que unifica sonido y significado de las expresiones lingüísticas y que puede servir como imput de otros sistemas de procesamiento mentales.

En un sentido muy básico, todos los sistemas cognitivos requieren estímulos perceptivos para poner en marcha sus operaciones. El sistema de procesamiento lingüístico, en particular, se activa a partir de estímulos auditivos (ondas sonoras) que constituyen las palabras. En este punto comienzan las características que singularizan al mecanismo lingüístico y que no tienen los otros sistemas de procesamiento. Las palabras activan el procesamiento de las representaciones más tempranas, las cuales pertenecen

1 Atkinson, A., "Wholes and their Parts in Cognitive Psychology: Systems, Subsystems, and Persons", http://www.soc.unitn.it/dsrs/IMC/IMC.htm, 1998.

2 Piccinini, G. y Craver. C., "Integrating Psychology and Neuroscience: Functional Analyses as Mechanism Sketches”, Synthese 183, 2011, pp. 283-311.

${ }^{3}$ Jackendoff, R., Foundations of Language, Oxford, OUP, 2002. 
a los sistemas de análisis auditivo y visual y tienen la función de extraer la información perceptiva del estímulo. ${ }^{4} \mathrm{La}$ particularidad que tiene el sistema de procesamiento lingǘstico propiamente dicho (y que no tienen los demás sistemas de procesamiento propiamente perceptivo) es que, una vez superado el análisis inicial realizado por los sistemas perceptivos de análisis lingüístico, el sistema lingüístico opera sobre representaciones que se activan por el solo funcionamiento de sus cómputos. Así, una vez activado, el sistema del lenguaje opera sobre un arreglo de información sintáctica, semántica, etc., de modo de dar lugar, como output, a una representación que unifica sonido y significado de una expresión. ${ }^{5}$

\footnotetext{
${ }^{4}$ Ellis, A. W. y Young, A. W., Neuropsicología cognitiva bumana, Barcelona, Masson, 1992.

${ }^{5}$ Chomsky, N. "Three Factors in Language Design", Linguistic Inquiry, Vol. 36, 1, 2005, pp. 1-22.
}

Fitch, W. T., Hauser, M. D., Chomsky, N., "The evolution of the language faculty: Clarifications and implications", Cognition, 97, 2005, pp. 179-210.

Hauser, M., Chomsky, N., y Fitch, W. T. "The language faculty: What is it, who has it, and how did it evolve?", Science, 298, 2002, pp. 1569-1579.

Lorenzo González, G., "El tercer factor: Reflexiones marginales sobre la evolución de la sintaxis", Teorema, Vol. XXV/3, 2006, pp. 77-92.

Pinker, S. y Jackendoff, R., "The faculty of language: what's special about it?", Cognition, 95, 2005, pp. 201-236.

El modelo de procesamiento de la información lingüística que presento hace énfasis en el reconocimiento lingüístico debido a que este aspecto del lenguaje será central en el argumento de la sección 3. También hay modelos del procesamiento de la información lingüística que se focalizan en la producción del lenguaje tales como el de Levelt (Speaking. From Intention to Articulation, Cambridge, MA, MIT Press, 1989). Cabe aclarar que entiendo la noción de procesamiento del lenguaje en relación a las nociones de "facultad del lenguaje" y "lengua-I" de la literatura minimalista. Siguiendo a Collins ("The Faculty Disputes", Mind and Language 19, 2004, pp. 503533): "la facultad del lenguaje debería ser vista como un conjunto de condiciones sui generis a las cuales responden las operaciones de un cerebro normal" (p. 4). Si se tiene en cuenta que el mecanismo que cumpliría con las condiciones abstractas sui generis de las que habla Collins, es el sistema de procesamiento de la información lingüística, entonces podría considerarse que la facultad del lenguaje, haciendo hincapié en el componente computacional de la lengua-I, es un módulo específico de la arquitectura cognitiva de la mente/cerebro encargado del procesamiento de la información lingüística. 
El procesamiento de la información lingüística, como otros sistemas de procesamiento, involucra representaciones mentales. Suele considerarse que las representaciones mentales pueden analizarse de acuerdo a dos aspectos: contenido y vehículo. El contenido es aquello acerca de lo que es la representación, es decir, aquello que es representado. En este sentido, se relaciona con las propiedades semánticas de las representaciones. El vehículo, en cambio, es el medio representacional a través del cual la representación representa algo, es decir, el formato que porta la información que lleva la representación. En este sentido, se relaciona con las propiedades no semánticas de las representaciones (denominadas, generalmente, sintácticas). ${ }^{6}$ Si el contenido y el vehículo constituyen aspectos bien diferenciados de las representaciones, entonces ¿cuál sería el vehículo de las representaciones del procesamiento de la información lingüística?

Siguiendo a Fodor, ${ }^{7}$ Schneider ${ }^{8}$ y Aydede, ${ }^{9}$ las representaciones del procesamiento del lenguaje estarían vehiculadas por un lenguaje diferente al lenguaje natural. Por ejemplo, las representaciones que intervienen en los procesos necesarios para hablar y comprender el español no se vehiculan en fórmulas del español. Por el contrario, pertenecen a un lenguaje interno que no es ningún lenguaje natural y que tiene como constituyentes mínimos a los conceptos. ${ }^{10}$ En sentido estricto, el lenguaje del pensamiento es una hipótesis acerca del formato de las representaciones que constituyen el contenido de los deseos, creencias y demás tipos de pensamientos. Pero, siguiendo a Fodor, Schneider y Aydede, la hipótesis del lenguaje del pensamiento también puede aplicarse al caso de las representaciones del procesamiento del lenguaje. El lenguaje del pensamiento del procesamiento de la información lingüística no tendría exactamente las mismas características que el lenguaje del pensamiento que subyace al pensamiento, pues no sería full blown, es decir, no

${ }^{6}$ Weiskopf, D., "Concept Empiricism and the Vehicles of Thought", The Journal of Consciousness Studies 14, 2007, pp. 156-183.

${ }^{7}$ Fodor, J., El lenguaje del pensamiento, Madrid, Alianza, 1975.

8 Schneider, S., "The Language of Thought", en P. Calvo y J. Simons (eds.) Routledge Companion to Philosophy of Psychology, Routledge, 2008.

${ }^{9}$ Aydede, M., "The Language of Thought Hypothesis", en E. Zalta (ed.) Stanford Encyclopedia of Philosophy, http://plato.stanford.edu/entries/language-thought, 2004.

${ }^{10}$ Fodor, J., Conceptos. Donde la ciencia cognitiva se equivocó, Barcelona, Gedisa, 1998. 
estaría completamente desarrollado, pero esto sería solamente una diferencia de grado. ${ }^{11}$

En este trabajo quisiera argumentar contra la idea de que el lenguaje del pensamiento es el formato de las representaciones del procesamiento de la información lingüística. En la primera parte evaluaré los denominados "argumentos del regreso" 12 con los que se ha intentado sostener la tesis escéptica de este trabajo. Concluiré que estos argumentos no constituyen una buena estrategia para desechar al lenguaje del pensamiento como vehículo representacional. En la segunda parte del trabajo, desarrollaré una "inferencia a la explicación menos probable" con la que considero se puede poner en duda el rol del lenguaje del pensamiento como formato de las representaciones del procesamiento del lenguaje. Es cierto que la hipótesis del lenguaje del pensamiento también puede ponerse en duda tomando en cuenta que, hasta lo que sé, parecería no haber evidencia neurobiológica de que el cerebro opere de acuerdo al tipo de representaciones simbólicas que caracterizan esta propuesta. Con todo, mi propósito en este trabajo es hacer una evaluación del lenguaje del pensamiento para el caso de las representaciones lingüísticas estrictamente en el plano de la psicología del procesamiento de la información. Tomando de manera independiente las explicaciones de esta área de la psicología, quisiera dar razones para preferir la hipótesis del lenguaje natural como vehículo de las representaciones del procesamiento del lenguaje.

11 En realidad, Schneider y Aydede consideran que el lenguaje del pensamiento también sería el vehículo de otros módulos como el del procesamiento de la información perceptiva. Con ello, se aceptaría que existen representaciones perceptivas que tendrían formato lingüístico, más puntualmente, del lenguaje del pensamiento. Sin embargo, en este trabajo no retomaré esta cuestión que está sujeta a debate.

12 Margolis. E. y Laurence S., "Regress arguments against the language of thought", Analysis 57, 1997, 60-66. Margolis E. y Laurence. S., "Where the Regress Argument Still Goes Wrong: Reply to Knowles”, Analysis 59, 1999, pp. 321-327. 


\section{Los argumentos del regreso}

Suele sostenerse que el lenguaje del pensamiento es un tipo de formato amodal y linguaforme de las representaciones. Es amodal, debido a que no pertenecen a ninguna modalidad perceptiva. ${ }^{13}$ Es linguaforme porque, de mínima, el lenguaje del pensamiento es un tipo de lenguaje entendido al modo de los lenguajes artificiales tales como los de la lógica o la matemática. ${ }^{14}$ Tiene propiedades como una sintaxis combinatoria y una semántica composicional. La sintaxis combinatoria consiste en que las representaciones moleculares tienen representaciones atómicas que respetan las relaciones parte-todo. ${ }^{15}$ Una representación mental compleja, por ejemplo, MAMA AMA A ULISES, está constituida por los constituyentes semánticos MAMA, AMAR y ULISES, que mantienen entre si ciertas relaciones estructurales. La semántica composicional consiste en que el contenido de la representación molecular depende del contenido de los constituyentes sintácticos atómicos y de sus relaciones estructurales. El aporte semántico de los constituyentes es independiente del contexto dado que cada constituyente realiza siempre la misma contribución al contenido de la representación molecular.

El lenguaje del pensamiento no es el único vehículo representacional amodal y lingüístico. También puede considerarse al lenguaje natural como un tipo de formato amodal y linguaforme de ciertas representaciones mentales. ${ }^{16}$ Esto lleva a considerar a la hipótesis del lenguaje del pensamiento en relación a la hipótesis alternativa según la cual el vehículo representacional es el lenguaje natural. Tal como se las presenta en la bibliografía, las hipótesis del lenguaje del pensamiento y del pensamiento en lenguaje natural son hipótesis acerca de los vehículos del pensamiento. El filósofo que más ha defendido la primera propuesta es Fodor y el que ha defendido la segunda propuesta de manera paradigmática es Carruthers. Por su parte, la hipótesis

${ }^{13}$ Carruthers, P. y Boterill, G., The Philosophy of Psychology, Cambridge, MA, MIT Press, 1999.

14 Werning, M., Machery, E. y Schurz, G., The Composicionality of Meaning and Content, vol. I., Frankfurt, Verlag, 2005.

15 Skidelsksy, L., Representaciones mentales. Donde la filosofía de la mente y la filosofía de la ciencia cognitiva se equivocaron, Buenos Aires. Eudeba, 2016.

16 Carruthers, P., Language, Thought and Consciousness, Cambridge, CUP, 1996. 
del pensamiento en lenguaje natural puede entenderse en un sentido fuerte y débil. De acuerdo al sentido fuerte, todo tipo de pensamiento se vehicula en oraciones del lenguaje natural por una cuestión de necesidad conceptual. De acuerdo al sentido débil, solamente los pensamientos proposicionales conscientes se vehiculan en oraciones del lenguaje natural, pero por una cuestión de necesidad natural. Más allá de sus diferencias, ambas hipótesis comparten la idea de que el vehículo del pensamiento es simbólico y, más exactamente, linguaforme.

Autores como Skidelsky ${ }^{17}$ tienden a sostener que en el estado actual de la investigación empírica y de los argumentos filosóficos, no hay manera de elegir cuál de estas hipótesis hace afirmaciones correctas respecto de nuestros pensamientos:

...considero que a pesar de que sería deseable poder dirimir la cuestión entre el lenguaje del pensamiento y el pensamiento en lenguaje natural para el caso de las mentes humanas -dado su interés no sólo para la investigación empírica acerca de nuestros mecanismos cognitivos y, en particular, para una teoría del pensamiento, sino para cuestiones filosóficas acerca de la relación entre lenguaje natural y pensamiento, que han acosado a los filósofos desde la antigüedad y que persisten en su carácter elusivo-, esto no parecería ser posible, al menos, con los recursos disponibles.

Esto sería así porque ambos tipos de vehículos son amodales y linguaformes, con lo cual, estarían en igualdad de condiciones para explicar casi las mismas propiedades del pensamiento (productividad, sistematicidad, entre otras). ${ }^{18}$ No pretendo hacer una evaluación concreta de las razones que

17 Skidelsky, L., “¿Lenguaje del pensamiento o pensamiento en lenguaje natural?”, en C. Celestino Silva y L. Salvatico (eds.), Filosofia e História da Ciência no Cone Sul, Porto Alegre, Entrementes Editorial, 2012, pp. 255-263.

${ }^{18}$ El pensamiento tiene propiedades como la productividad y la sistematicidad. Es productivo porque hay una cantidad potencialmente infinita de pensamientos que se pueden tener, cada uno de los cuales es diferente de manera arbitraria en su contenido y estructura (Fodor, J., "Having Concepts: a Brief Refutation of the Twentieth Century", Mind \& Language 19, 2004, 29-47). A pesar de las restricciones que de facto impone nuestra actuación, en principio, podemos generar un número infinito de pensamientos. Además, el pensamiento es sistemático porque la habilidad de producir o entender un pensamiento está intrínsecamente conectada con la habilidad de 
llevarían a sostener que no hay manera de decidir entre estas hipótesis en el marco del estudio de las propiedades del pensamiento. No obstante, entiendo que atender a sus similitudes no es una manera fructífera de encarar el estudio de la hipótesis del lenguaje del pensamiento frente a la del lenguaje natural. Esta sería una manera incorrecta de evaluar al lenguaje del pensamiento como vehículo del procesamiento del lenguaje que puede identificarse en los argumentos del regreso.

Existe una serie de argumentos en contra del lenguaje del pensamiento en el procesamiento del lenguaje que Margolis y Laurence han denominado "argumentos del regreso". Son tres argumentos que tienen la forma de un dilema y que comienzan tomando en consideración ciertas propiedades de los lenguajes naturales. El primero de ellos parte de la idea de que los lenguajes naturales son comprendidos, el segundo parte de la idea de que los lenguajes naturales son aprendidos y el tercero parte de la idea de que las oraciones del lenguaje natural tienen significado. No quiero detenerme en ninguno de ellos en particular, solamente quisiera destacar que todos responden a la misma estructura que, siguiendo a Margolis y Laurence, puede resumirse de la siguiente manera:

(1) El lenguaje natural tienen cierta propiedad $x$ (el lenguaje natural es comprendido, es aprendido, sus oraciones son significativas).

(2) Los defensores del lenguaje del pensamiento apelan a un sistema de representaciones internas para explicar esa propiedad de los lenguajes naturales. El lenguaje del pensamiento también tiene la propiedad $x$.

(3) Esto da lugar al siguiente dilema: si ofrecemos una explicación de la propiedad $x$ del lenguaje del pensamiento, en términos de otro lenguaje (tal como se hace al explicar la propiedad $x$ del lenguaje natural), entonces se generaría una regresión infinita de lenguajes. Si se da una explicación alternativa de por qué el lenguaje del pensamiento tiene la propiedad $x$, entonces se debería haber dado esa misma explicación para dar cuenta de la

producir o entender otros pensamientos (Fodor, J. y Pylyshyn, Z. W., "Connectionism and Cognitive Architecture: a Critical Analysis", en C. McDonald y G. McDonald (eds.) (1995) Connectionism, Oxford, Blackwell, 1988) Si una persona piensa que Juan ama a la niña, también tiene que poder pensar que la niña ama a Juan. Es una cuestión empírica acerca de cómo son nuestras capacidades lingüísticas que existan ciertas simetrías en el poder expresivo en el sistema lingüístico humano. 
propiedad $x$ que tiene el lenguaje natural, evitando así postular un lenguaje del pensamiento.

(4) Por lo tanto, el lenguaje del pensamiento no explica la propiedad $x$ del lenguaje natural.

Puede entenderse este esquema de argumento interpretando a $x$ como la propiedad que tienen los lenguajes naturales de ser comprendidos. Esta propiedad de los lenguajes naturales es posible porque hay un mapeo que va de las expresiones del lenguaje natural a las expresiones del lenguaje del pensamiento. Si la comprensión de los lenguajes naturales requiere el mapeo del lenguaje natural a las expresiones del lenguaje del pensamiento y, a su vez, el lenguaje del pensamiento es comprendido, entonces ¿en qué consiste la comprensión de las expresiones del lenguaje del pensamiento? Claramente, no puede sostenerse que esta comprensión consiste en un mapeo de las expresiones del lenguaje del pensamiento a las expresiones de otro lenguaje porque nos obligaría a sostener un regreso infinito de lenguajes. Entonces, debería ser posible comprender las expresiones del lenguaje del pensamiento sin necesidad de ningún mapeo. Pero si se puede comprender las expresiones del lenguaje del pensamiento sin necesidad de hacer ningún mapeo en otro lenguaje, entonces también podría darse la comprensión de las expresiones del lenguaje natural sin necesidad de mapearlas en las expresiones de un lenguaje interno.

Aunque la bibliografía presenta este tipo de argumentos como un ataque a la hipótesis del lenguaje del pensamiento aplicada al pensamiento, creo que también podría presentarse como un argumento que apoya la idea de que los procesos del lenguaje natural no operan sobre representaciones del lenguaje del pensamiento. Si atendemos al segundo cuerno del dilema, vemos que este tipo de argumentos abre la posibilidad de que ciertas propiedades de los lenguajes naturales (ser comprendidos, ser aprendidos, ser significativos) se expliquen sin necesidad de postular ningún mapeo a las expresiones de otro lenguaje que no sea el natural.

Margolis y Laurence sostienen que la fuerza de este tipo de argumentos se sostendría junto con otros argumentos que ataquen otras razones para sostener el lenguaje del pensamiento. Visto con su propia luz, la fuerza del argumento del regreso depende de que haya otros argumentos contra toda la gama de razones positivas para adherir al lenguaje del pensamiento. 
Solamente ahí el argumento del regreso tendría fuerza y en ese caso, de todas maneras, ya no sería necesario. Lejos de "destruir" al lenguaje del pensamiento, el argumento del regreso ofrece poca o nula razón para ser escépticos respecto de esta hipótesis.

Por mi parte, entiendo que el argumento del regreso debería desestimarse por la razón de que parte del supuesto de que el lenguaje del pensamiento comparte una serie de propiedades con los lenguajes naturales. $\mathrm{Y}$ es cierto que muchas de las propiedades que tiene el lenguaje del pensamiento son las que tiene el lenguaje natural (sistematicidad, productividad, entre otras). Pero la analogía entre lenguaje natural y lenguaje del pensamiento no puede ir tan lejos. El lenguaje del pensamiento no siempre comparte todas las mismas propiedades del lenguaje natural. Esta es la idea que incorrectamente se introduce en la premisa (2). Por ejemplo, cuando se dice que el lenguaje natural se "comprende" y se dice que el lenguaje del pensamiento se "comprende", se está usando dos nociones distintas de "comprensión”. En palabras de Fodor:

Hay dos maneras en las que decimos que un dispositivo (incluida presumiblemente una persona) comprende un predicado. En un caso, el dispositivo tiene y usa una representación de la extensión del predicado, donde la representación es ella misma dada en algún lenguaje que el dispositivo comprende. En el segundo caso, el dispositivo está construido de tal manera que su uso del predicado (e.g., en computaciones) se acomoda a las condiciones que esa representación especificaría. Lo primero es verdad para los predicados del lenguaje natural que la gente aprende y lo segundo es verdad para los predicados de un lenguaje interno. ${ }^{19}$

Si hay nociones distintas de "comprensión", entonces el lenguaje del pensamiento no tiene la propiedad $x$ del lenguaje natural, según la cual, en este caso, los lenguajes naturales se comprenden. Si el lenguaje del pensamiento no tiene la propiedad $x$ que tiene el lenguaje natural, es decir, si se quiebra la analogía entre ambos lenguajes, entonces no puede darse lugar al dilema presentado en la premisa (3).

${ }^{19}$ Fodor, J., El lenguaje del pensamiento, p. 66. 
Los argumentos del regreso, los cuales se basan en las similitudes entre el lenguaje del pensamiento y el lenguaje natural, nos muestran que para elegir entre la hipótesis del lenguaje natural o la del lenguaje del pensamiento habría que atender más bien a las diferencias entre estas hipótesis. A pesar de sus similitudes, la hipótesis del lenguaje del pensamiento tiene características que no tiene la hipótesis del lenguaje natural, y estas características la volverían provisionalmente menos fructífera. Esto es lo que exploraré en la siguiente sección con una inferencia a la explicación menos probable.

\section{Una inferencia a la explicación menos probable}

El argumento que desarrollo en esta sección, en contra de que el procesamiento de la información lingüística opere sobre representaciones del lenguaje del pensamiento, tiene la estructura de lo que denominaré una "inferencia a la explicación menos probable" y puede resumirse en los siguientes pasos:

(1) Tómese el fenómeno de la eficacia en el reconocimiento léxico.

(2) Se lo puede explicar, en parte, o bien apelando a representaciones de un lenguaje del pensamiento o bien apelando a representaciones del lenguaje natural.

(3) Entre estas explicaciones rivales, la menos probable es la que apela a representaciones de un lenguaje del pensamiento.

(4) Por lo tanto, es menos probable que sea correcta la explicación que apela a representaciones de un lenguaje del pensamiento.

Los psicolingüistas suelen aceptar que nuestra remarcable capacidad de comprender el lenguaje hablado depende, en gran medida, de la eficacia con la que podemos reconocer las palabras en nuestro léxico mental. ${ }^{20}$ Así, la eficacia es el fenómeno según el cual el procesamiento subyacente al reconocimiento léxico genera un output lingüístico recién cuando se cuenta con toda la información lingüística necesaria y con independencia de la información no

20 Kolinksy, R., Morais, J. y Segui, J., La Reconnaissance des Mots dans les Differentes Modalités Sensorielles, Paris, Presses Universitaires de France, 1991. 
lingüística. Este fenómeno parece rescatar la idea de Fromkin ${ }^{21}$ según la cual la naturaleza de las representaciones determina en parte la naturaleza de los mecanismos léxicos. Así, la manera de concebir las representaciones tendría repercusiones a la hora de dar cuenta de los procesos.

Si se admite que el reconocimiento oral de las palabras consiste en una proyección del sonido a los conceptos y los conceptos son representaciones mentales, entonces tiene que haber algún tipo de intervención de representaciones en los procesos que subyacen a la comprensión de las palabras habladas. ${ }^{22}$ Si la eficacia es un fenómeno de los procesos de comprensión oral de las palabras y si, a su vez, estos procesos operan sobre representaciones, entonces es muy probable que la eficacia en la comprensión oral de las palabras se relacione con estas representaciones propias del sistema lingüístico.

Hay dos grandes maneras de entender los vehículos de las representaciones del procesamiento del lenguaje. ${ }^{23} \mathrm{La}$ primera describe la posición de Fodor y otros. El procesamiento del lenguaje opera sobre representaciones vehiculadas por el lenguaje del pensamiento. La segunda es la posición que sostiene que el procesamiento del lenguaje opera sobre representaciones vehiculadas por el lenguaje natural. Si lo que propongo es una inferencia a la explicación menos probable, cabe hacerse la siguiente pregunta: ¿cuál de estas opciones es la que está en peores condiciones para explicar la eficacia en la comprensión oral de las palabras? La comprensión

${ }^{21}$ Fromkin, E., Speech Errors as Linguistic Evidence, La Haya, Mouton, 1973.

22 Belichón Carmona, M., Igoa Gonzáles y J. M., Riviere Gómez, A., Psicología del lenguaje. Investigación y teoría, Madrid, Trotta, 1998. Marslen-Wilson, W. D. "Acceso e integración: La proyección del sonido sobre el significado”, en W. D. Marslen-Wilson (ed.), Lexical Representation and Procesess, Cambridge, MA, MIT Press, 1989.

23 El enfoque dominante en el estudio del procesamiento del lenguaje natural es el que apela a representaciones linguaformes. No pretendo desarrollar enfoques alternativos, como el conexionista, donde las computaciones lingüísticas se basan en continuos matemáticos de redes neuronales (Smolensky, P, "Conectionist Approaches to Language”, en R. A. Wilson y F. C. Keil (eds.), The MIT Encyclopedia of the Cognitive Sciences, Cambridge, MA, MIT Press, 2001). No está del todo claro que el conexionismo sea realmente un enfoque alternativo y, aun cuando lo sea, creo que todavía no ha logrado tener el poder explicativo que tienen las posiciones que apelan a representaciones con vehículos de formato lingüístico. 
oral de las palabras es eficaz en tanto que el output lingüístico se genere tomando en cuenta la información lingǘstica (fonética, semántica o sintáctica) necesaria para dicha comprensión. Esta información lingüística puede estar en el formato del lenguaje natural o del lenguaje del pensamiento. Pero esta última opción sería deficitaria a la luz de la primera.

La razón de esto se relaciona con la adopción de un criterio metodológico de elección entre explicaciones posibles. Ese criterio es el de la simplicidad y puede expresarse de la siguiente manera: Si E es más simple que $\mathrm{E}^{*}$, entonces es racional adoptar E (como explicación). ${ }^{24}$ Podemos reformular este principio de la siguiente manera: $\mathrm{Si}^{*}$ es menos simple que $\mathrm{E}$, entonces es racional no adoptar $\mathrm{E}^{*}$. Si interpretamos a $\mathrm{E}^{*}$ como la explicación que plantea representaciones vehiculadas por el lenguaje del pensamiento y a $\mathrm{E}$ como la explicación que plantea representaciones vehiculadas por el lenguaje natural, entonces se puede afirmar, por simplicidad, que la propuesta de Fodor tiene menos probabilidad de explicar la eficacia. En lo que sigue analizaré en qué sentido la propuesta de Fodor es menos simple que la otra opción.

Cualquier computadora digital se caracteriza por tener un sistema de compiladores. Estos compiladores son algoritmos de traducción que convierten los imputs en un formato que pueda ser comprensible en el lenguaje de máquina. La función de los compiladores es traducir a representaciones de un lenguaje interno, solamente accesible a la máquina, la información que viene contenida en los inputs que recibe la computadora. Fodor considera que en las capacidades lingüísticas humanas (entre las cuales está incluida la comprensión oral de las palabras) también intervienen una serie de compiladores. Si el lenguaje del pensamiento que subyace a las capacidades lingüísticas vendría dado en el organismo del mismo modo que toda computadora está construida con su propio lenguaje de máquina y si el lenguaje de máquina exige un sistema de compiladores que traduzcan los inputs a representaciones computables por el lenguaje de máquina, entonces

24 Baker, A. "Simplicity", Standford Encyclopedia of Philosophy (edición primavera de 2003), E. N. Zalta (ed.), http://plato.stanford.edu/entries/simplicity/, 2010. 
también las capacidades lingüísticas humanas tienen que tener este sistema de compiladores. ${ }^{25}$

Puede pensarse que Fodor utiliza la noción de "compilador", para el caso de las capacidades lingüísticas humanas, de dos maneras. Primero, afirma que la traducción se da del input físico al lenguaje interno del pensamiento y viceversa: “... [los] compiladores hacen posible que el hablante/oyente traduzca de las fórmulas del código computacional a las fórmulas ondulatorias y viceversa."26 Aquí "código computacional" significa lenguaje del pensamiento y "fórmulas ondulatorias" significa input físico. Segundo, afirma que la traducción se da de las oraciones del lenguaje natural a las oraciones del lenguaje del pensamiento y viceversa: “....es posible y provechoso pensar que un compilador que asocia cada una de las fórmulas del lenguaje de input I con fórmulas en el lenguaje de computación $C$ es el metalenguaje en que se representan las propiedades semánticas de las oraciones de $I . " 27$

La primera noción de "compilador" no sería problemática. La comprensión oral de las palabras involucra una serie de procesos mentales para lo cual es necesario traducir el estímulo físico (las ondas acústicas) a un medio representacional mental, sea éste el que propone el defensor del lenguaje del pensamiento u otro. Fodor considera que la traducción se da de las fórmulas ondulatorias al lenguaje del pensamiento y viceversa, pero podría sostenerse que la traducción se da de las fórmulas ondulatorias a un sistema de representaciones internas (sean o no del lenguaje del pensamiento) y viceversa.

La otra noción de "traducción", en cambio, surge de la adopción de la hipótesis del lenguaje del pensamiento. En la comprensión, hay una traducción que va del lenguaje natural al lenguaje del pensamiento. Por ejemplo, para comprender la palabra "perro", hay un/unos compilador/es que traduce/n esta representación léxica a una representación PERRO del lenguaje del pensamiento. Cuando nos preguntamos por los detalles de este tipo de traducción nos encontramos con un proceso que es altamente

\footnotetext{
25 Ver Fodor (La modularidad de la mente, Madrid, Morata, 1983) para las semejanzas y diferencias entre la noción de "compilador" y la noción de "transductor".

${ }^{26}$ Fodor, J., El lenguaje del pensamiento, p. 131.

${ }^{27}$ Ibid., p. 134
} 
misterioso. ¿Qué quiere decir exactamente que “...un compilador $[. .$.$] asocia$ cada una de las fórmulas del lenguaje de imput I con fórmulas en el lenguaje de computación C..."?28 ¿Significa traducir las representaciones léxicas de $I$, que tienen el formato de un lenguaje natural a las representaciones de $C$, que tienen el formato de un lenguaje del pensamiento? Si esto es así, ¿cómo puede llevarse a cabo el pasaje de un código representacional a otro? Además, si el proceso de traducción involucra un pasaje de las fórmulas de $I$ a las fórmulas de $C$, ¿cómo garantizar que las fórmulas de $C$ expresan la misma información lingüística que las fórmulas de $I$ ? En verdad, hay una manera de evitar todas estas complicaciones. Si consideramos que las representaciones que subyacen a la comprensión oral de las palabras son del lenguaje natural y que, por esta razón, PERRO, por ejemplo, es una representación vehiculada por lenguaje natural, entonces no necesitaríamos apelar a ningún tipo de compilación.

La eficacia propia de los procesos de comprensión léxica se vería obstaculizada más que simplificada si se adopta la explicación del lenguaje del pensamiento, pues habría una instancia de traducción que va de representaciones del lenguaje natural a representaciones del lenguaje del pensamiento que conspiraría contra la simplicidad de la explicación del fenómeno de la eficacia. Esta instancia de traducción no existe en el caso de proponer representaciones vehiculadas en lenguaje natural. Dado que la propuesta de Fodor es menos simple que su competidora, en tanto que incorpora una instancia de traducción que no está en esta última, entonces está en inferioridad de condiciones para explicar el fenómeno de la eficacia.

En realidad, la propuesta de Fodor de representaciones lingüísticas en formato del lenguaje del pensamiento no sólo es infructuosa para el caso de la explicación del fenómeno de la eficacia. También tendría dificultades para explicar otros fenómenos estudiados por la psicolingüística. La hipótesis del lenguaje del pensamiento afecta de manera directa a fenómenos como el priming semántico, la rapidez de los procesos, entre otros. El priming consiste en una disminución en los tiempos de reacción frente a un estímulo, de modo que es un fenómeno íntimamente relacionado con la rapidez de los procesos de comprensión lingüística. ${ }^{29}$ La rapidez, por su parte, es el fenómeno según el cual el procesamiento subyacente a la comprensión lingüística genera un

\footnotetext{
${ }^{28}$ Ibid.

${ }^{29}$ Valle Arroyo, F., Psicolingüistica, Madrid, Morata, 1992.
} 
output lo más cercano temporalmente al estímulo. La rapidez es un fenómeno diferente al de la eficacia, pero puede decirse que son las dos caras de una misma moneda en el sentido de que son las dos características fundamentales de los procesos de comprensión lingüística. El procesamiento que subyace a la comprensión que genera un output cuando se cuenta con toda la información lingüística suficiente y satisfactoria no tiene un tiempo indefinido, sino que, por el contrario, genera este output con la mayor celeridad posible. El compromiso simultáneo entre la eficacia y la rapidez es lo que determina el éxito que de hecho manifestamos en la comprensión de las palabras. De la misma manera que la eficacia, la rapidez se ve afectada por la postulación de un vehículo representacional del lenguaje del pensamiento, dado que un tipo de vehículo que exige un paso adicional en los procesos retarda, de alguna manera, la generación del output. Así, si el priming está íntimamente relacionado con la rapidez y la rapidez se ve directamente obstaculizada por la postulación de un vehículo en lenguaje del pensamiento, entonces también el priming se ve directamente obstaculizado por la postulación de un vehículo en lenguaje del pensamiento.

La inferencia a la explicación menos probable también podría proponerse para el caso del priming semántico. En este caso también sería necesario postular un nivel computacional de traducción que va de las representaciones del lenguaje natural a las representaciones del lenguaje del pensamiento, lo cual volvería más compleja la explicación de los fenómenos en cuestión. Así, también en los casos del priming semántico la explicación más sencilla sería considerar que los procesos operan sobre representaciones del lenguaje natural y no sobre representaciones del lenguaje del pensamiento. En este caso, se evitaría incorporar un nivel de procesamiento innecesario para dar cuenta satisfactoriamente de estos fenómenos.

Una objeción podría ser la siguiente. Quizá es cierto que la propuesta del lenguaje del pensamiento es menos simple que su rival para dar cuenta del fenómeno de la eficacia (una propone un mecanismo de traducción innecesario en la otra), y que esto sea una razón para abandonarla. Pero ¿por qué creer que la hipótesis del lenguaje del pensamiento tiene que explicar esto? ¿Por qué creer que la eficacia en la comprensión de las palabras habladas forma parte del conjunto de fenómenos que la hipótesis fodoriana tiene que explicar? Esta objeción tendría una respuesta. La eficacia es un fenómeno 
empírico que atañe al procesamiento del lenguaje. La propuesta de Fodor de que hay representaciones del lenguaje del pensamiento es, primero, una hipótesis postulada para muchos dominios cognitivos, pero también es acerca del procesamiento de la información lingüística y, segundo, es una hipótesis empírica. Por eso es esperable que a la luz de esta hipótesis se explique la eficacia en la comprensión léxica. De hecho, Fodor ${ }^{30}$ (1998) propone el "argumento de la carga del procesamiento" mediante el cual concluye que las representaciones del lenguaje del pensamiento no tienen una estructura interna de información (razón por la cual son representaciones atómicas) teniendo como objetivo explicar ciertos fenómenos empíricos del procesamiento léxico, como la rapidez.

La idea de que las representaciones del procesamiento del lenguaje se vehiculan en oraciones del lenguaje natural se apoyaría en un argumento que, a diferencia de los argumentos del regreso, revela la diferencia que existe entre el lenguaje natural y el lenguaje del pensamiento. A diferencia de la hipótesis del vehículo del lenguaje natural, la hipótesis del lenguaje del pensamiento nos compromete con cuestiones como la siguiente: "¿qué aspectos de la interpretación de la expresión forman parte de la facultad del lenguaje, qué otros aspectos pertenecen a la semántica del lenguaje natural?"31 Por su parte, la hipótesis del vehículo en lenguaje natural permite evadir este tipo de planteos problemáticos.

\section{Conclusión.}

Fodor afirma que:

El carácter sintáctico del pensamiento sugiere una visión de los procesos cognitivos en general-incluso, por ejemplo, la percepción, la memoria y el aprendizaje - que propone que estos se podrían dar en un medio de naturaleza lingüística, en una suerte de lenguaje del pensamiento. ${ }^{32}$

${ }^{30}$ Fodor, J., Conceptos. Donde la ciencia cognitiva se equivocó, Barcelona, Gedisa, 1998.

31 Chomsky, N., La arquitectura del lenguaje, Barcelona, Kairós, 2003, p. 4.

32 Fodor, J., El olmo y el experto, Barcelona, Paidós, 1997, p 25. 
Siguiendo el espíritu de la cita, puede interpretarse que la hipótesis del lenguaje del pensamiento también se aplica a las representaciones computadas en el procesamiento del lenguaje. Esto es lo que sugieren autores como el mismo Fodor, Aydede y Schneider. Frente a ello, la hipótesis de que el procesamiento del lenguaje opera sobre representaciones vehiculadas por el lenguaje natural sería mejor porque no incorporaría mecanismos de compilación que pueden obstaculizar la explicación de fenómenos como la eficacia en la comprensión léxica, entre otros. Así, al menos en el caso del procesamiento del lenguaje puede pensarse que la hipótesis del vehículo en lenguaje natural es más fructífera que la hipótesis del lenguaje del pensamiento.

La inferencia a la explicación menos probable presentada en la sección anterior, con unas pocas modificaciones, puede convertirse en una inferencia a la mejor explicación:

(1) Tómese el fenómeno de la eficacia en el reconocimiento léxico.

(2) Se lo puede explicar, en parte, o bien apelando a representaciones de un lenguaje del pensamiento o bien apelando a representaciones del lenguaje natural.

(3) Entre estas explicaciones rivales, la mejor es la que apela a representaciones del lenguaje natural.

(4) Por lo tanto es más probable que sea correcta la explicación que apela a representaciones del lenguaje natural.

La conclusión, en este caso, también está posibilitada por el criterio de la simplicidad. La explicación según la cual los procesos de comprensión del lenguaje operan sobre las representaciones del lenguaje natural no necesita incorporar los mecanismos de compilación propuestos por Fodor. En este sentido, las representaciones que subyacen al reconocimiento de las palabras tendrían vehículos propios del lenguaje natural. Por supuesto que "la ciencia cognitiva está en su infancia” y que quizá el futuro desarrollo pueda ayudar a una mejor descripción de los símbolos del lenguaje del pensamiento. ${ }^{33}$ Pero

33 Schneider, S., "The Central System as a Computational Engine”, en The Language of Thought: A New Philosophical Direction, Cambridge, MA, MIT Press, 2011. 
lo que importa es el estado actual de la investigación. Y si atendemos al estado actual de la investigación, creo que hay al menos una razón para sostener la hipótesis del lenguaje natural por sobre la del lenguaje del pensamiento en el caso de las representaciones del procesamiento del lenguaje.

Recibido: 06/2017; aceptado: 11/2017 